\title{
Motivationsschreiben im Deutschen: Eine textlinguistische Betrachtung
}

[The German Motivationsschreiben: a textlinguistic analysis] http://dx.doi.org/10.1590/1982-8837198215

Franziska Schwantuschke ${ }^{1}$

\begin{abstract}
This study aims to analyse the text genre "Motivationsschreiben", a document that must be prepared by applicants who aspire joining in a master's program or a scholarship in Germany. Based on a corpus of 30 "Motivationsschreiben" which were collected for the dissertation from the University of Leipzig and UFPR between February and July 2014 the analysis focused on the relevance, the communicative context and functions of the text genre.
\end{abstract}

Keywords: Application; Text genre; Communicative functions; Communicative context

Resumo: $O$ presente trabalho tem como objetivo analisar o gênero textual "Motivationsschreiben", documento que deve ser elaborado por candidatos que almejam o ingresso num programa de mestrado ou uma bolsa de estudos na Alemanha. Baseando-se em um corpus de 30 "Motivationsschreiben" recolhidos para a dissertação na Universidade de Leipzig e na UFPR entre os meses de novembro de 2013 a maio de 2014, a análise enfocou a relevância, o contexto comunicativo e as funções do gênero textual.

Palavras-chaves: Candidatura; Gênero textual; Funções comunicativas; Contexto comunicativo

Zusammenfassung: Ziel des vorliegenden Artikels ist die Analyse von Motivationsschreiben, einer Textsorte, die bei der Bewerbung um einen Masterstudienplatz oder um ein Stipendium häufig eingereicht werden muss. Anhand eines Korpus von 30 Motivationsschreiben, welche im Rahmen der Masterarbeit von November 2013 bis Mai 2014 gesammelt wurden, sollen die Relevanz, der Kommunikationskontext und die Textfunktionen der offenen Textsorte aufgezeigt werden.

Stichwörter: Bewerbung; Textsorte; Textfunktion; Kommunikationskontext

\footnotetext{
${ }^{1}$ Universität Leipzig, Herder Institut; Universidade Federal do Paraná, Setor de Ciências Humanas, Letras e Artes, Binationaler Masterstudiengang Deutsch als Fremdsprache. Email: franziska@schwantuschke.com
} 


\title{
1 Einleitung
}

\begin{abstract}
Hallo liebe Master - Studenten! Ich möchte mich für das kommende Wintersemester für den Masterstudiengang DaF bewerben. Kann mir jemand sagen, was für das Motivationsschreiben gefordert wird? Hat jemand eine kurze Hilfestellung für mich parat? Vielen Dank im Voraus!(FACEBOOK-EINTRAG vom 21.4.2014)
\end{abstract}

Verfasst ein Bewerber zum ersten Mal ein Motivationsschreiben, steht er vor der großen Frage, was ein solches Schreiben eigentlich erfassen sollte. Dass damit selbst muttersprachliche Studierende philologischer Fächer Probleme haben, zeigt der Hilferuf.

Unter den Bewerbungstextsorten wurden zu den Textsorten Lebenslauf (vgl. dazu u.a. HEPP 2006, MORELlo 2006) und Vorstellungs- bzw. Bewerbungsgespräch (vgl. dazu u.a. LEPSCHY 1995, SCHMIDT 2000, B IRKNER 2001, SCHILLING 2001) bereits zahlreiche linguistische Studien sowohl aus einzelsprachlicher als auch kontrastiver Perspektive durchgeführt. Außerdem existieren empirische Untersuchungen $\mathrm{zu}$ den Textsorten Anschreiben (vgl. dazu SCHRÖDER 2002) und Stipendienantrag (vgl. dazu KOTTHOFF 2009).

Bezüglich der Textsorte Motivationsschreiben liegen m. E. noch keine empirisch fundierten wissenschaftlichen Arbeiten vor; nur praktisch orientierte Ratgeber in Form von vereinzelten Büchern, vor allem aber als digitale Online-Versionen sind vorhanden. Auch Freudenberg-Findeisen weist in ihrer Rezension zu Fandrychs und Thurmairs „Textsorten des Deutschen“ darauf hin, dass ,[a]us sprachdidaktischer Perspektive und das zeigen Unterrichtspraxis wie auch Lehrwerke - [...] »offenere « Textsorten wie journalistischer Bericht, Reportage, Umfrage, Motivationsschreiben sehr wünschenswert [wären]““ (FREUDENBERG-FINDEISEN 2011: 574).

Der folgende Artikel fasst einige Ergebnisse meiner Masterarbeit „Motivationsschreiben im Deutschen. Empirische Analyse und didaktische Implikationen für den DaF-Unterricht“ zusammen. Für diese Arbeit wurde von Februar bis Juli 2014 ein Korpus von 30 Motivationsschreiben, die im Rahmen einer Studienoder Stipendienbewerbung von deutschen Muttersprachlern verfasst wurden, erstellt. Hinsichtlich des Studienfachs wurden keine Beschränkungen vorgenommen. Die meisten Korpusexemplare kommen aber aus dem geisteswissenschaftlichen Bereich. 


\section{Texte, Textsorten, Textmuster}

Textsorten entwickeln sich als Lösungsstrategien für spezifische kommunikative Aufgaben, welche aufgrund von sich verändernden kommunikativen Bedürfnissen in einer Gesellschaft aufkommen (vgl. KRAUSE 2000: 48). Mit der Zeit entwickeln und festigen sich standardmäßige Ausprägungen, sogenannte Textmuster. Sie werden gelernt und reproduziert. Durch die ständige Reproduktion erfährt das Muster Differenzierung und Variation, wodurch die Textsorte auf ihre Funktionserfüllung hin überprüft werden muss (vgl. GANSEL 2011: 110). BRINKERS umfassende Textsortendefinition, die auch von Textmustern ausgeht, soll grundlegend für den weiteren Verlauf angenommen werden (1997: 132):

Textsorten sind konventionell geltende Muster für komplexe sprachliche Handlungen und lassen sich als jeweils typische Verbindungen von kontextuellen (situativen), kommunikativ-funktionalen und strukturellen (grammatischen und thematischen) Merkmalen beschreiben. Sie haben sich in der Sprachgemeinschaft historisch entwickelt und gehören zum Alltagswissen der Sprachteilhaber; sie besitzen zwar eine normierende Wirkung, erleichtern aber zugleich den kommunikativen Umgang, indem sie den Kommunizierenden mehr oder weniger feste Orientierung für die Produktion und Rezeption von Texten geben.

Textsorten beziehen sich auf konkrete Realisierungen von Texten, die das Auftreten atypischer Merkmale nicht ausschließen (vgl. FELD-KNAPP 2005: 123). Besonders Textsorten mit einem geringeren Standardisiertheitsgrad lassen eine größere Variation unter den einzelnen Textexemplaren zu. Deswegen umfasst ein Textmuster nur idealtypische Modelle, in denen ,[...] atypische Modelle keinen Platz haben“ (ebd.). Textmuster dienen zur Identifikation und Zuordnung eines Textexemplars zu einer Textsorte. Textexemplare können ,[...] erhebliche Unterschiede aufweisen, nicht nur, was die konkrete sprachliche Ausgestaltung, sondern auch, was ihre Textstruktur betrifft (Anordnung und Vorkommen von Teiltexten etwa)“ (THURMAIR 2001: 271). Deswegen sollten beim Fremdsprachenunterricht vor allem Texte zum Einsatz kommen, die als prototypische Vertreter einer Textsorte gelten (vgl. VENOHR 2007: 28ff.).

Hinsichtlich der Auswahl der im Unterricht zu behandelnden Textsorten fordert THIM- MABREY: „Studierende sollten ein Textsortenwissen erwerben, das ihnen dazu verhilft, diejenigen Textsorten, in denen sie selbst schriftliche Texte produzieren, kompetent in Einzeltexten zu realisieren“" (2005: 34). 


\section{Gesellschaftliche Relevanz}

Spätestens in der Ausbildungs- bzw. Studienphase und/oder zum Übergang in die Arbeitswelt, konkret in der Bewerbungsphase, muss man sich mit der Textsorte Motivationsschreiben auseinandersetzen. Dabei ist nur die Produktion, im seltensten Falle auch die Rezeption, relevant. Die Texthandlung, die durch die Rezeption des Motivationsschreibens vom Adressaten vollzogen wird, kann entweder eine Ablehnung oder eine Befürwortung des Bewerbers sein. Werden die Konventionen der Bewerbungsunterlagen nicht eingehalten, wird dies im schlimmsten Fall mit Ausschluss der Bewerbung sanktioniert.

Der Bewerber versucht sich demnach aus eigenem Interesse an die Konventionen zu halten. Eine schlichte Nichtbenutzung/Ablehnung der Textsorte, sollte diese als Teil der Bewerbungsunterlagen ausdrücklich gefordert sein, wird in der Regel ebenso sanktioniert, da Bewerber mit inkompletten Bewerbungsmappen nicht in die nähere Auswahl kommen.

Die Forderung nach Motivationsschreiben dehnt sich auf verschiedenste Bereiche aus: Nicht nur im Rahmen der Berufs-, Studien- und Stipendienbewerbung werden Motivations- schreiben gefordert, sondern auch bei der Bewerbung um einen Studentenwohnheimplatz, der Teilnahme an Workshops und Wettbewerben, der Projektförderung, der Tätigkeit als Bildungsmentor und Kirchenführer. Dass das Motivationsschreiben allerdings (noch) kein selbstverständlicher Bestandteil der Berufsund Studienbewerbungsunterlagen ist, zeigen die zwei folgenden Ausschnitte aus Ausschreibungen:

(1) Bewerbungen müssen schriftlich mit den üblichen Unterlagen sowie einem Motivationsschreiben bei der DIU eingereicht werden. (LOGISTIK INSIDE 2009: 59)

(2) Für den [Tüftler-Wettbewerb, Anm. der Verfasserin] können sich alle Schüler der Klassen 9 bis 13 bewerben: Übrigens nicht mit ihren Zeugnissen, sondern mit einem Motivationsschreiben. (LÜKE 2011: 22)

Dennoch wird das Motivationsschreiben zur Eignungsfeststellung immer üblicher und einflussreicher. Bei einer Masterbewerbung am Herder-Institut der Universität Leipzig kommt dem Motivationsschreiben zum Beispiel eine Gewichtung von 20\% der Gesamtbewerbung zu (vgl. Herder-Institut 2014). In den meisten Bewerbungsratgeberbüchern findet das Motivationsschreiben gar keine Erwähnung. 


\section{Schwantuschke, F. - Motivationsschreiben}

Umso größer ist die Unwissenheit der Bewerber über Inhalt und Form. Das Abschreiben von Mustermotivationsschreiben aus dem Internet oder aus Ratgebern kann Inhaltsarmut und fehlende Individualität zur Folge haben. Weil das Motivationsschreiben eine sehr gering standardisierte Textsorte ist, ist es dem Bewerber möglich, durch Individualität und Kreativität zu überzeugen, indem er sich ,,[...] von 'Floskeln' lös[t] und persönliche und individuelle Formulierungen [...] wähl[t]““ (FANDRYCH/THURMAIR 2011: 317).

\section{Einbettung der Textsorte}

Die Textsorte Motivationsschreiben ist unter die Textsortengruppe der Vorstellungstexte zu fassen. FANDRYCH und THURMAIR definieren diese als ,„[...] Texte, mit denen sich eine Person selbst in einer öffentlichen oder halböffentlichen Situation vorstellt $[\ldots]^{“ /}(2011: 36)^{2}$.

Textsorten stehen nicht verbindungslos nebeneinander. Sie „bilden ein Gesamtsystem, innerhalb dessen sie einen bestimmten Platz und Stellenwert haben“ (ADAMZIK 2007: 16). FANDRYCH und THURMAIR nennen dieses Gesamtsystem Textverbund. Ein Textverbund umfasst ,[...] verschiedene Textsorten(exemplare), die in strukturierter Anordnung räumlich zusammen auftreten (und häufig auf spezifische Weise aufeinander bezogen sind)“ (2011: 27). Das Motivationsschreiben ist ein Teil des Textverbundes Bewerbungsunterlagen. Im Handlungszusammenhang der Bewerbung tritt es gemeinsam mit den Komplementärtexten Lebenslauf, Anschreiben und Anlagen auf. Zusammen mit seinen Komplementärtexten ist das Motivationsschreiben in einer „syntagmatischen Textsortenkette“ zu verorten (vgl. ADAMZIK 2007: 27). Diese entsteht, wenn ein Text andere Texte nach sich zieht oder voraussetzt. Folgende syntagmatische Textsortenkette kann für die komplexe Handlung Bewerbung angenommen werden:

\footnotetext{
${ }^{2}$ Wie in 5 näher erläutert, handelt es sich bei der Bewerbungskommunikation nicht um eine öffentliche, sondern eine offizielle Korrespondenz. Demnach kann parallel zu der Auffassung, dass der formelloffizielle Lebenslauf ein Vorstellungstext ist, auch das Motivationsschreiben als solcher angesehen werden (vgl. FANDRYCH/THURMAIR 2011: 37).
} 


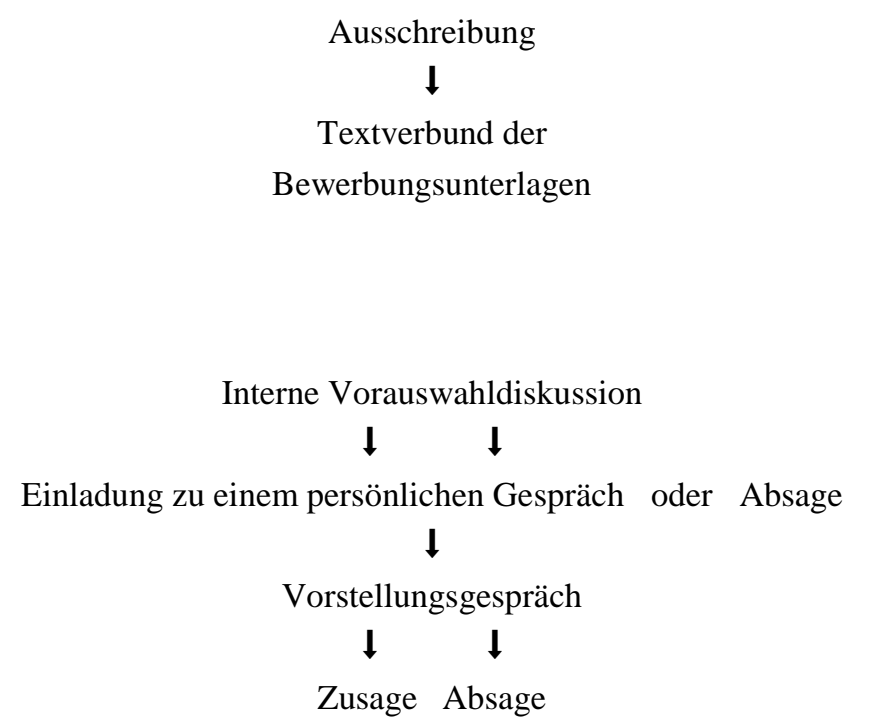

Die Ausschreibung entfällt bei einer Initiativbewerbung.

Die Unterlagen setzen sich üblicherweise aus Anschreiben, Lebenslauf, Motivationsschreiben und Zeugnissen, Gutachten und Zertifikaten als Anlagen zusammen.

Bei manchen Bewerbungsprozessen entfällt das Vorstellungsgespräch (z.B. einige Masterbewerbungen). Es kann aber auch durch weitere Instrumente der Bewerberselektion (z.B. AssessmentCenter) erweitert werden.

Die syntagmatische Kette (siehe Abbildung) ist in ihrer Abfolge obligatorisch. Beispielsweise kann der Ablehnungsbescheid nicht vor der Einreichung der Bewerbungsunterlagen versandt werden. Wie die Hinweise neben der Abbildung zeigen, müssen nicht in jedem Bewerbungsprozess alle Schritte vorhanden sein. Die Texte stehen in einem größeren Zusammenhang zueinander. KLEIN (2000) prägt den Begriff der Textsorten-Intertextualität (ebd.: 33$)^{3}$.

Wie SCHMIDT (2000) in seiner Darstellung der sprachlichen und nichtsprachlichen Handlungen eines Bewerbungsprozesses darstellt, sind die Kontaktschritte abwechselnd auf Bewerber- und Entscheidungsträgerseite angesiedelt. Bei der Erstellung der Bewerbungsunterlagen (ein interner Prozess seitens des Bewerbers) versucht der Bewerber bestmöglich auf den von der Institution erstellten Kriterienkatalog, der ihm nicht bekannt ist, zu reagieren. Der interne Kriterienkatalog besteht vermutlich aus Richtlinien und erwarteten Textsortenkonventionen, die für den Bewerber nicht transparent sind. Bei der Bewertung der Motivationsschreiben gehen die Rezipienten von „[...] ,stillschweigende[n] Regeln' der Stellenvergabe und Bewerberauswahl [aus], die nirgends gesetzlich oder vertraglich verankert sind“ (REIß/VERMEER 1991: 184 nach MORELLO 2006: 59). Selbst auf Nachfrage bei renommierten Institutionen, die Stipendien und Studienplätze vergeben, wurden keine

\footnotetext{
${ }^{3}$ Deutlich wird die Textsorten-Intertextualität bei Betrachtung des expliziten Bezugs der Betreffzeile einesAnschreibens auf ein ausgeschriebenes Stellenangebot.
} 


\section{Schwantuschke, F. - Motivationsschreiben}

Aussagen über die Richtlinien zum Verfassen des gewünschten Motivationsschreibens gemacht. Dabei unterstreicht BOLTEN, dass ,[z]ur besseren Strukturierung der Auswahl die Anforderungen an das Motivationsschreiben so explizit formuliert werden [sollten], dass für BewerberInnen eine eindeutige Orientierungsvorgabe besteht" (2013: $5)$.

\section{Kommunikationskontext}

Relevant wird das Textsortenwissen über Motivationsschreiben bei Neu- oder Umorientierung in biografischen Umbruchsituationen, in denen eine Bewerbung verfasst wird. Das Motivationsschreiben muss immer im Kontext des Textverbundes Bewerbung betrachtet werden. Aus Datenschutzgründen müssen die Bewerbungsunterlagen vertraulich behandelt und der Öffentlichkeit vorenthalten werden, d.h. sie zählen zur nicht-öffentlichen Kommunikation (vgl. MoRELLO 2006: 80). Kennzeichnend für die zudem offizielle Korrespondenz zwischen dem Bewerber und der Institution ist der ,[...] bei weitem höhere[ ] Grad an Verbindlichkeit als im privaten Bereich“" (BRINKER 1997: 137).

\subsection{Kommunikationsbereich}

Kommunikationsbereiche etablieren sich dadurch, dass Menschen in ihren bestimmten Handlungsrollen kommunikativ handeln, indem sie Texte produzieren und rezipieren (vgl. GANSEL 2011: 12). ADAMZIK spricht sich gegen solche „Ordnungsgröße für die Verortung von Textsorten“ aus (vgl. 2004: 73). Deutlich wird ihr Einwand, wenn man versucht, die Textsorte Motivationsschreiben einem Kommunikationsbereich zuzuordnen. Textsorten, die einem Kommunikationsbereich zugeordnet werden können, werden in dessen Rahmen sozial und situativ definiert und folgen den dort geltenden Handlungsnormen (vgl. FANDRYCH/THURMAIR 2011: 17).

Wie eingangs ausgeführt, können Motivationsschreiben $\mathrm{zu}$ unterschiedlichen Zwecken verfasst werden. Demnach kann die Textsorte sowohl den Kommunikationsbereichen Hochschule (z. B. im Rahmen einer Masterbewerbung), Schule (z.B. im Rahmen einer Praktikumsbewerbung), Wirtschaft und Handel (z.B. im Rahmen einer Berufsbewerbung), als auch Alltag (z. B. im Rahmen einer Bewerbung um einen Wohnheimplatz) zugeordnet werden. Des Weiteren ist das 
Motivationsschreiben unabhängig vom Zweck dem Kommunikationsbereich Verwaltung zugehörig.

Die vorliegende Untersuchung betrachtet nur Motivationsschreiben, die im Kommunikationsbereich Hochschule zum Zweck der Studien- und Stipendienbewerbung verfasst wurden.

\subsection{Kommunikationssituation}

Es handelt sich bei einem Motivationsschreiben um einen medial schriftlichen Text. Kennzeichnend für medial schriftliche Texte ist die fehlende Kopräsenz von Leser und Schreiber und dass das Kommunikat räumlich und zeitlich getrennt vom Rezeptionsmoment verfasst wird (vgl. FANDRYCH/THURMAIR 2011: 18). So liegt normalerweise eine zeitliche Verzögerung zwischen dem Erstellen und Versenden durch den Produzenten und dem Rezipieren und Antworten durch den Rezipienten vor. Die nicht-simultane Kommunikation (räumliche und zeitliche Trennung des Produktions- und Rezeptionsprozesses) wirkt sich auch auf die Gestaltung der Textsorte aus.

Das Verbreitungsmedium der Bewerbungsunterlagen (einschließlich des Motivationsschreibens) hat sich mit den modernen Medien verändert: Verschickte man vor ein paar Jahren die Bewerbungsunterlagen noch in Briefform mit der Post, ist das Versenden per E- Mail heutzutage weitaus verbreiteter. Die Bewerbungsunterlagen werden einer E-Mail im Anhang (meist im pdf-Format) beigefügt. Außerdem verfügen einige Institutionen (z.B. Auswärtiges $\mathrm{Amt}^{4}, \mathrm{DAAD}^{5}$ ) über Bewerberportale. Mit einem Account, den sich der Bewerber eigens für den Bewerbungsprozess anlegt, kann er die geforderten Unterlagen hochladen oder den Text des Motivationsschreibens direkt in eine Maske eingeben. Mithilfe der Maske kann der Rezipient die Zeichen- oder Wortanzahl automatisch begrenzen. Außerdem erkennen die Portale automatisch, wenn die Bewerbungsunterlagen unvollständig abgeschickt werden.

\footnotetext{
${ }^{4}$ Vgl. https://service.diplo.de/praktikanten_ausland/bewerbung.php (07/08/2014)

${ }^{5}$ Vgl. https://portal.daad.de/irj/portal (07/08/2014) 


\subsection{Kommunikationspartner}

Produzent des Motivationsschreibens ist normalerweise der Bewerber selbst ${ }^{6}$. Er modelliert sich seinen idealtypischen Leser und konstruiert beim Produktionsprozess das Wissen, das er von diesem erwartet. Adressat ist die Institution, bei der man sich bewirbt, oder deren Vertreter. Die Anzahl der Kommunikationspartner variiert von einer Eins-zu-Eins-Kommunikation bis hin zu einer Eins-zu-Mehreren-Kommunikation. Je nachdem wie viele Personen über die Zulassung eines Bewerbers entscheiden, fällt die Anzahl der Rezipienten aus ${ }^{7}$. Eine angenommene Mehrfachadressiertheit zeigt sich durch die standardisierte Begrüßungsformel sehr geehrte Damen und Herren.

Normalerweise herrscht zwischen dem Verfasser und dem Leser eine „soziale Distanz“, da der Leser nicht bekannt ist (vgl. HARTING 2010: 403). Der Grad der Kooperativität ist sehr gering, da die Statusbeziehung zwischen den Kommunikationspartnern durch ein Hierarchie-Gefälle charakterisiert ist. Der Rezipient ist ein Vertreter einer höheren Instanz, was ihn dazu befugt, über die Einstellung des Bewerbers zu urteilen ${ }^{8}$. In der hierarchisch niedrigeren Position strebt der Bewerber danach, den Anforderungen an ihn zu entsprechen (vgl. MORELLO 2006: 83).

\subsection{Produktions- und Rezeptionsprozess}

Das Motivationsschreiben ist eine monologische, medial schriftliche und somit asynchrone Textsorte. Aufgrund der Einseitigkeit der Kommunikation muss der Text aussagekräftige und für die Stelle relevante Informationen enthalten und diese müssen logisch strukturiert sein, um verstanden zu werden. Das Motivationsschreiben weist ein geringes Maß an Responsivität auf, da eine Rückkopplung nur bedingt möglich ist. Das heißt, dass der Bewerber beim Produktionsprozess auf eine schlüssige Argumentation und eine thematisch vielfältige Selbstdarstellung achten muss, da aufgrund der einseitigen Kommunikation keine Rückfragen oder näheren Erklärungen auf inhaltlicher Ebene an ihn herangetragen werden (können). Der Rezipient kann höchstens im Falle eines Vorstellungsgesprächs noch einmal auf eventuelle Lücken

\footnotetext{
${ }^{6}$ Allerdings gibt es auch professionelle Schreiber, die ihre Dienste über das Internet anbieten, z. B. unter http://www.motivationsschreiben.de/. (08/10/2014)

${ }^{7}$ BOLTEN plädiert dafür, dass am Auswahlprozess mehrere „Staff“-Mitglieder beteiligt sein sollten, um eine „Blickwinkelvielfalt“"zu gewährleisten (vgl. 2013: 5).

${ }^{8}$ Da es sich um einen „statushöheren Leser“ handelt, spricht HARTING von „Macht“ (vgl. 2010: 403).
} 
oder nähere Erläuterungen eingehen.

Der Produktionsvorgang nimmt damit sehr viel mehr Zeit in Anspruch als der Rezeptionsprozess. Abhängig von der Geübtheit, der Anzahl der bereits verfassten Motivationsschreiben und der Bewerbungsfrist kann der Produktions- und Revisionsprozess eines Motivationsschreibens zeitlich variieren. Die Erstellung eines Motivationsschreibens sollte für jede Bewerbung neu erfolgen, wobei sich die Phase der Textproduktion auf das Aufarbeiten und Umformulieren eines bereits abgefassten Dokuments beschränken kann. Ob die Textrezeption partiell, vollständig, ein- oder mehrmalig erfolgt, ist vom Rezipienten abhängig (vgl. MORELLO 2006: 80).

\section{Textfunktion}

Die Textfunktion ist ,[...] die im Text mit bestimmten, konventionell geltenden, d.h. in der Kommunikationsgemeinschaft verbindlich festgelegten Mitteln ausgedrückte Kommunikationsabsicht des Emittenten“ (BRINKER 1997: 93). Sie stellt sowohl die Intention des Textproduzenten als auch die potentielle Erwartung des Textrezipienten dar (vgl. HeINEMANN 2001: 304). Die Textfunktion ist zudem eng verbunden mit der thematischen Struktur?.

Das am weitesten verbreitete Textfunktionsmodell von Brinker unterscheidet in Anlehnung an die illokutiven Grundfunktionen der Searle'schen Sprechakttheorie ${ }^{10}$ fünf grundlegende Funktionstypen: die Informationsfunktion, die Appellfunktion, die Obligationsfunktion, die Kontaktfunktion und die Deklarationsfunktion. Die Textfunktionen bündeln die Textsorten in Textsortenklassen. Die Textsortenklassifikation nach Funktionstypen basiert auf der dominierenden Funktion der Textsorte. In manchen Textfunktionsmodellen werden neben den fünf Textfunktionen nach Brinker zusätzliche Funktionen, wie die werbende, die selbstdarstellende, die unterhaltende oder auch die ästhetische Funktion angenommen. Letztere bezieht sich ausschließlich auf die fiktionale Welt literarischer Texte. Ihre Existenz ist umstritten (vgl. FANDRYCH/THURMAIR 2011: 19).

\footnotetext{
${ }^{9}$ Diese Abhängigkeit drückt BRINKER im Kompatibilitätsprinzip aus: „Als Hauptthema des Textes ist [...] das Thema zu betrachten, das sich am besten mit der aufgrund einer textpragmatischen Analyse ermittelten Textfunktion verträgt“"(1997: 56f.).

${ }^{10}$ In seiner Sprechakttheorie nimmt SEARLE die fünf Sprechakttypen der Assertiva, Direktiva, Kommissiva, Expressiva und Deklarativa an. Anders als in Brinkers Textfunktionsmodell beschränkt sich Searle in seiner Theorie auf die Satzebene.
} 
BRINKER geht von einer Polyfunktionalität der Textsorten aus. Das heißt, dass ein Text mehrere Funktionen innerhalb einer Kommunikationssituation haben kann, wobei meist eine dominiert. Deswegen unterscheidet man zwischen der Textfunktion (dominierende Funktion) und den Nebenfunktionen (vgl. BRINKER 1997: 81ff.).

Die Textfunktion bestimmt wesentlich die sprachliche Ausgestaltung eines Textes. Ihre Bestimmung leitet sich deswegen ,[...] aus dem Wechselspiel der konkreten empirischen Textsortenanalyse und der Analyse ihrer typischen kommunikativen und gesellschaftlichen Einbettung und Zwecksetzung ab [...]“ (FANDRYCH/THURMAIR 2011: 33). VENOHR geht sogar davon aus, dass ,[...] bei der Beschreibung von Text(sort)en [...] alle Textsortenmerkmale in Abhängigkeit zur Textfunktion [stehen]“" (2007: 77).

Sprachliche Handlungen, die im Rahmen der Sprechakttheorie Illokutionen genannt werden, werden im Text intentional geäußert, das heißt, dass jede sprachliche Handlung eine Funktion im Text hat (vgl. BRINKER 1997: 86). BRINKER nennt drei „Illokutionsindikatoren“, die zur Identifikation der dominierenden Textfunktion dienen können (vgl. ebd.: 97f.):

1) Sprachliche Formen und Strukturen, mit denen der Emittent die Art des intendierten kommunikativen Kontakts dem Rezipienten gegenüber explizit zum Ausdruck bringt.

2) Sprachliche Formen und Strukturen, mit denen der Emittent - explizit oder implizit - seine Einstellungen zum Textinhalt, insbesondere zum Textthema ausdrückt.

3) Kontextuelle Indikatoren wie der situative, insbesondere der institutionelle Rahmen des Textes bzw. der gesellschaftliche Handlungsbereich, dem der Text zugeordnet ist, das vorausgesetzte Hintergrundwissen (z.B. über den Textinhalt) usw.

Textfunktionen werden durch sprachliche Handlungen und Vertextungsstrategien ausgedrückt. Da jede sprachliche Handlung in einen Handlungs- bzw. Situationszusammen- hang eingebettet ist, müssen Kontextindikatoren (z. B. das Rollenverhältnis, der institutionelle Rahmen und das Hintergrundwissen) berücksichtigt werden (vgl. BRINKER 1997: 89). BRINKER geht davon aus, dass die sprachlichen Indikatoren unzureichend oder unverlässlich sein können, weswegen letztendlich die kontextuellen Indikatoren entscheidend sind (vgl. ebd.: 97). FANDRYCH und THURMAIR (2011: 20) schließen sich dieser Beobachtung an: 
Dafür [zur Analyse der Textfunktion, Anm. der Verfasserin] ist die Einbeziehung typischer Kommunikationskonstellationen, der Produktions- und Rezeptionssituationen, des institutionellen Rahmens, des thematischen Bezugs und der Handlungseinbettung ebenso wichtig wie die Analyse der Textstruktur und der typischerweise in den Texten realisierten sprachlichen Handlungen.

\subsection{Dominierende Textfunktion}

Auf das Anschreiben und den Lebenslauf folgend kann der Bewerber mit dem Motivationsschreiben sowohl inhaltliche Unklarheiten des Lebenslaufs aufklären als auch seine „rhetorische Kompetenz“ demonstrieren (vgl. SCHRÖDER 2002: 175):

Der Bewerber kann [...] die Rezeption seiner Bewerbung steuern und die Aufmerksamkeit des Lesers lenken, er kann Aspekte der formalen oder realen Qualifikationen besonders markieren oder in der Relevanz zurückstufen, scheinbare oder tatsächliche biographische Brüche erklären bzw. verständlich machen oder insgesamt die Rezeption der Bewerbung erleichtern.

Die Einschätzung des „Handlungs- und Argumentationspotentials“ des Bewerbers ist besser über das Motivationsschreiben als über das Anschreiben möglich, da es ausführlicher und weniger formelhaft ist. Dennoch ist auch das Motivationsschreiben im Umfang begrenzt (meist zwei Seiten). Der Bewerber muss sich für Informationen aus seinem Lebenslauf entscheiden, die er für die Tätigkeit förderlich findet (Selektion des Inhalts ${ }^{11}$. Die Fähigkeit, eine komplexe schriftliche Kommunikationssituation angemessen $\mathrm{zu}$ bewältigen, und die rhetorische Strukturierungsleistung werden unter Beweis gestellt (vgl. ebd.: 156).

Als Subtextsorte des Textverbundes Bewerbung übernimmt das Motivationsschreiben dessen übergeordnete Funktion des Werbens (vgl. MoRELLO 2006: 42). Die werbende Funktion wird als primäre Textfunktion nicht direkt, sondern durch ,indirekte Sprechakte“ realisiert. Mithilfe ausgewählter Informationen soll der Leser von der Eignung des Bewerbers überzeugt werden. Dazu führt er nur Qualifikationen und erfüllte Voraussetzungen an, die für ihn werben.

\footnotetext{
${ }^{11}$ Die Selektion des Inhalts hat außerdem eine assertive Intention als Hintergrund: Die gezielte Informationsvermittlung bewegt den Leser dazu, etwas Bestimmtes (den gewählten Inhalt) zur Kenntnis zu nehmen, zu wissen, zu glauben und/oder zum Gegenstand seiner eigenen Einstellung zu machen (vgl. SÄNDIG 2005: 157).
} 


\section{Schwantuschke, F. - Motivationsschreiben}

Aus der Vermittlung der ausgewählten Informationen ergibt sich zudem die Funktion der Wissensvermittlung. Diese liegt vor, wenn ein Ungleichgewicht an Wissen zwischen der Sender- und Empfängerseite existiert (vgl. LÖFFLER 2007: 81). Wird das bereitgestellte Wissen vom Rezipienten allerdings „,nicht umstandslos akzeptiert“, muss mit „rational nachvollziehbaren zusätzlichen Wissenselementen“ Akzeptanz hergestellt werden (FANDRYCH/THURMAIR 2011: 30). Daraus ergibt sich eine argumentativwerbende, wissensvermittelnde Funktion als Textfunktion der Textsorte Motivationsschreiben. Die wissensvermittelnde Funktion kann in verschiedenen Realisationsformen ausgedrückt werden. Man unterscheidet die sachbetonte, die meinungsbetonte und die emotive Realisationsform. Welche Realisationsform vorliegt, lässt sich anhand sprachlicher Mittel erkennen (vgl. THIM-MABREY 2005: 37). Bei den Motivationsschreiben variiert die Darstellung der Informationen von einer meinungsbetonten zu einer emotiven Realisationsform.

\subsection{Kontaktfunktion}

Bei der Kontaktfunktion gibt ,,[d]er Emittent [...] dem Rezipienten zu verstehen, daß es ihm um die personale Beziehung zum Rezipienten geht [...]“ (BRINKER 1997: 119). Anders als bei Brinkers Beschreibung des Funktionstyps handelt es sich bei einem Motivations- schreiben nicht um einen „Kontakttext ${ }^{“}$ zu einem „festen gesellschaftlichen Anlass“ oder die „Erfüllung einer sozialen Erwartung“(vgl. ebd.). Dennoch wird der Leser in einigen Textexemplaren direkt kontaktiert. Sprachlich spiegelt sich die kontaktierende Textfunktion zum einen in der Adressatendeixis (Sie, Ihnen, Ihrerseits) und zum anderen im Gebrauch von Begrüßungs- und Abschiedsformeln wider.

Einerseits wird durch den direkten Kontakt die Individualität der Institution unterstrichen (Beleg 1 und 2) und gezeigt, dass ein persönliches Kennenlernen gewünscht ist (Beleg 3 und 4):

(1) [...] deshalb bei Ihnen um den dort ausgeschriebenen Erasmus- Platz im Wintersemester 12/13 zubewerben. (AS_11) ${ }^{12}$

(2) da ich an Ihrer Universität beste Voraussetzungen sehe, meinem beruflichen Ziel der BBB einen Schritt näher zu kommen. (MS_23)

\footnotetext{
${ }^{12}$ Die Angabe der Korpusbelege erfolgt durch Nummerierung und Zweck (AS = Auslandsstudium, $\mathrm{BS}=$ Bachelorstudium, $\mathrm{MS}=$ Masterstudium, $\mathrm{SA}=$ Sprachassistenz) .
} 


\section{Schwantuschke, F. - Motivationsschreiben}

(3) Über eine Einladung zu einem Gespräch, in welchem ich Ihnen persönlich meine hohe Motivation schildern kann, würde ich mich sehr freuen. (SA_28)

Andererseits hat die direkte Kontaktaufnahme auffordernden Charakter. Dem Leser wird vor Augen geführt, dass er die soziale Rolle des Entscheidungsträgers übernimmt:

(4) [ich] hoffe auf eine baldige Antwort Ihrerseits. (SA_29)

(5) [ich] würde mich über eine positive Antwort Ihrerseits auf meine Bewerbung sehr freuen. (MS_23)

Besonders deutlich wird die Appellfunktion durch die direkte Kontaktaufnahme in folgendem Beispiel:

(6) Wenn ich den von mir favorisierten Studienplatz für SSS erhalte, können Sie eine zielstrebige, verantwortungsbewusste und motivierte Studentin erwarten. (MS_25)

Durch den konditionalen Konnektor wenn wird ein Handlungsvorschlag ausgedrückt. Der Institution wird verdeutlicht, dass sie nur von dem Bewerber profitieren wird, wenn er die Zusage zum Studienplatz erhält.

\subsection{Appellfunktion}

Im Kapitel zur Kontaktfunktion wurde bereits der enge Zusammenhang zur Appellfunktion dargestellt. Besonders im letzten Beispiel ist die Appellfunktion dominierender als die Kontaktfunktion. Der Bewerber gibt dem Leser eine indirekte Handlungsorientierung. Der Rezipient soll dazu bewegt werden, eine bestimmte Einstellung bzw. Bewertung gegenüber dem Bewerber anzunehmen. Der Bewerber will damit den Bewerbungs- und Auswahlprozess beeinflussen.

Der Appell wird nicht direkt formuliert. Es lassen sich im Korpus keine Imperativformen oder Modalverben wie müssen oder sollen, die den Leser zu einer direkten Handlung aufrufen, finden. Allerdings soll mithilfe der argumentativen Vertextungsstrategie, bei der Thesen und Argumente formuliert werden, der Leser überzeugt werden.

\subsection{Obligationsfunktion}

Mit der Formulierung von Plänen und Zielen kündigt der Bewerber zukünftige 


\section{Schwantuschke, F. - Motivationsschreiben}

Handlungen an. Durch die Inaussichtstellung eigener Handlungen grenzt er seinen zukünftigen Handlungsspielraum ein. Die Obligationsfunktion in Motivationsschreiben bezieht sich auf den Sprecher selbst und ist daher eine „obligativsprecherbezogene Funktion“ (FANDRYCH/THURMAIR 2011: 31). Es ist sehr unwahrscheinlich, dass nach Beendigung einer erfolgreich verlaufenden Bewerbungsphase die Erfüllung versprochener Handlungen überprüft wird.

Auch die Appellfunktion und die Obligationsfunktion stehen in direkter Verbindung zueinander:

(7) Wenn ich den von mir favorisierten Studienplatz für SSS erhalte, können Sie eine zielstrebige, verantwortungsbewusste und motivierte Studentin erwarten. (MS_25)

Die konditionale Äußerung besteht aus zwei Teilen. Im ersten Teil des Satzes wird dem Leser eine Handlung vorgeschlagen. Der zweite Teil ist ein Handlungsversprechen seitens des Schreibers. Der Schreiber wird seine Selbstverpflichtung nur einlösen (können), wenn der Leser der vorgeschlagenen Handlung folgt.

Die Obligationsfunktion wird im Korpus außerdem durch sprachliche Mittel voluntativer und intentionaler Art verbalisiert. „Der Emittent kann dadurch den Grad an Bereitschaft ausdrücken, den thematisierten Sachverhalt zu realisieren“ (BRINKER 1997: 118). Besonders das futurische Prädikat in der ersten Person stellt eine Ankündigung dar, mit der sich der Sprecher zu einer zukünftigen Handlung verpflichtet:

(8) [...] und werde mich deshalb in nächster Zeit, neben meiner Bachelorarbeit, dem Verfassen eines sogenannten (Textsorte) widmen. (AS_04)

(9) Im Rahmen des (Projektname) werde ich versuchen, vor Ort Lernerdaten zu erheben. (SA_30)

(10) Ich werde darauf achten, keine "Fachidiotin" zu werden. (BS_16)

\section{Schlussbemerkung}

Die textlinguistische Beschreibung kann gewinnbringend für die Vermittlung des Deutschen als Fremdsprache genutzt werden. Die Behandlung der Textsorte Motivationsschreiben im DaF-Unterricht hat einen direkten sozialen Nutzen, da die sprachliche Handlung des Bewerbens in einer Fremdsprache durch eine international orientierte Studiensuche und durch Austauschprogramme an Relevanz gewinnt. 
Die Charakterisierung der Textsorte Motivationsschreiben hat gezeigt, dass Textsorten nicht isoliert voneinander betrachtet werden können. Die Einbettung in den Kommunikations- kontext und die Intertextualität zu anderen Textsorten haben einen großen Einfluss auf die Textfunktionen der Textsorte, die wiederum die sprachliche Gestaltung bestimmen. Den Lernenden muss der Gebrauchskontext und die Relevanz der Textsorte verdeutlicht werden. Die Einbettung der Textsorte in die Bewerbungshandlung und die Funktion und der Zweck der Subtextsorte Motivationsschreiben sollten vom Lehrenden sehr klar aufgezeigt werden.

Das Textthema des Motivationsschreibens ist für Lernende aller Niveaustufen zugänglich. Als einziges textsortenkonstitutives Merkmal ist die eigene Person als Textthema das wichtigste Merkmal der Textsorte. In den ersten Stunden des Fremdsprachenunterrichts steht immer die Vorstellung der eigenen Person im Vordergrund. Zudem erlaubt der geringe Standardisiertheitsgrad eine Variation der Textsorte auf inhaltlicher und struktureller Ebene.

\section{Literaturverzeichnis}

ADAMZIK, K. Textlinguistik. Eine einführende Darstellung. Tübingen, Niemeyer, 2004. ADAMZIK, K. Die Zukunft der Text(sorten)linguistik: Textsortennetze, Textsortenfelder, Textsorten im Verbund. In: FIX, U./ HABSCHEID, S./ KLEIN, J. (Hrsg.): Zur Kulturspezifik von Textsorten. Tübingen, Stauffenburg, 2007, 15-32.

BIRKNER, K. Bewerbungsgespräche mit Ost- und Westdeutschen. Eine kommunikative Gattung in Zeiten gesellschaftlichen Wandels. Tübingen, Niemeyer, 2001.

BOLTEN, J. Studierende als Experten für interkulturelle Handlungsfelder identifizieren, motivieren und fördern. http://www2.uni-jena.de/philosophie/IWKneu/typo3/fileadmin/team/juergen.bolten/1206Diversity_Experten_ik_Handlungsfelde r.pdf. (07/08/2014)

BRINKER, K. Linguistische Textanalyse. Eine Einführung in Grundbegriffe und Methoden.Berlin, Schmidt, 1997.

FANDRYCH, C.I THURMAIR, M. Textsorten des Deutschen. Linguistische Analyse aus sprachdidaktischer Sicht. Tübingen, Stauffenburg, 2011.

FELD-KNAPP, I. Textsortenspezifische Merkmale und ihre Relevanz für Spracherwerbsprozesse (DaF). In: ADAMZIK, K./ KRAUSE, W. (Hrsg.): TextArbeiten. Textsorten im fremd- und muttersprachlichen Unterricht an Schule und Hochschule. Tübingen, Narr, 2005, 115-135.

FREUDENBERG-FINDEISEN, R. Rezension. Fandrych, Christian; Thurmair, Maria. Textsorten im Deutschen. Linguistische Analysen aus sprachdidaktischer Sicht. In: Info DaF, München 2011, v.5, 572-575.

GANSEL, C. Textsortenlinguistik. Göttingen, Vandenhoeck \& Ruprecht, 2011.

HARTING, A. Textmuster von E-Mail-Bitten in der Fremdsprache Deutsch: Einfluss 


\section{Schwantuschke, F. - Motivationsschreiben}

mutter- und zielsprachlicher Textmuster. In: Info DaF, München 2010, v.4, 401-416.

HEINEMANN, W. Textsorten der geschriebenen Sprache. In: HELBIG, G. et al. (Hrsg.): Deutsch als Fremdsprache. Ein internationales Handbuch. Berlin u.a., de Gruyter, 2001, 300-313.

HEPP, M. Die Textsorte „Lebenslauf“ in interkultureller Perspektive. Anhaltspunkte für einen linguistisch fundierten universitären Daf-Unterricht. In: Studi Linguistici e Filologici Online, 2006, v.4.1, 109-130. http://www.humnet.unipi.it/slifo/index.html (09.06.2014).

HERDER-INSTITUT: http://www.uni-leipzig.de/herder/hi.site,postext,studium,a_id,4935.html (31.03.2014).

KLEIN, J. Intertextualität, Geltungsmodus, Texthandlungsmuster. Drei vernachlässigte Kategorien der Textsortenforschung - exemplifiziert an politischen und medialen Textsorten In: ADAMZIK, K. (Hrsg.): Textsorten. Reflexionen und Analysen. Tübingen, Stauffenburg, 2000, 31 - 44.

KOTTHOFF, H. Positionierungen in Stipendienanträgen: Zur interkulturellen Pragmatik einer akademischen Gattung. In: Info DaF, München 2009, v. 6, 483-499.

KRAUSE, W. Text, Textsorte, Textvergleich. In: ADAMZIK, K. (Hrsg.): Textsorten. Reflexionen und Analysen. Tübingen, Stauffenburg, 2000, 45-76.

LEPSCHY, A. Das Bewerbungsgespräch. Eine sprechwissenschaftliche Studie zu gelingender Kommunikation aus der Perspektive von Bewerberinnen und Bewerbern. St. Ingbert, Röhrig, 1995.

LÖFFLER, D. Eine Analyse von Textsorten in verschiedenen Formaten von „Ratgeberzeitschriften“. Greifswald, Masterarbeit, 2007. http://www.phil.unigreifswald.de/fileadmin/mediapool/philologie/ArbeitLoeffler.pdf (09.06.2014).

LOGISTIK INSIDE Master-Studiengang startet wieder im April. In: Logistik inside, 2009, v.3, 59.

LÜKE, G. Ausbildung - Individualität gewinnt. In: ProFirma, 2011, v.3: 22-32.

MORELLO, C. Die Textsorte Lebenslauf aus kontrastiver Sicht. Dargestellt am Deutschen und Französischen. Hamburg, Kovač, 2006.

REIß, K. / VERMEER, H. J. Grundlegung einer allgemeinen Translationstheorie. Linguistische Arbeiten, Bd. 147, 2. Auflage. Niemeyer, Tübingen, 1991.

SÄNDIG, U. Die Textsortenvariante 'literturwissenschaftlicher Kurzessay'. In: ADAMZIK, K./ KRAUSE, W. (Hrsg.): Text-Arbeiten. Textsorten im fremd- und muttersprachlichen Unterricht an Schule und Hochschule. Tübingen, Narr, 2005, 137154.

SCHILLING, A. Bewerbungsgespräche in der eigenen und fremden Sprache Deutsch. Empirische Analysen. Frankfurt am Main, Lang, 2001.

SCHMIDT, U. Bewerbung und Vorstellungsgespräch aus dialoglinguistischer Sicht. Einige Vorbemerkungen zur Aufarbeitung eines von der Linguistik vernachlässigten Arbeitsgebietes. In: Linguistik online, 2000, v.5. http://www.linguistikonline.de/1_00/Schmidt.htm (09.06.2014).

SCHRÖDER, P. Exemplarische Analyse eines Bewerbungsbriefs - Ein Vorschlag zur Handlungsschemaanalyse eines schriftlichen Kommunikationsereignisses. In: BECKER- MROTZEK, M./ FIEHLER, R. (Hrsg.): Unternehmenskommunikation. Tübingen, Narr, 2002, 145-178.

THIM-MABREY, C. Stilnormen als Textsortennormen. Korrektur und Beratung zu Texten von Schülern und Studierenden: In: ADAMZIK, K./ KRAUSE, W. (Hrsg.): TextArbeiten. Textsorten im fremd- und muttersprachlichen Unterricht an Schule und Hochschule. Tübingen, Narr, 2005, 33-45.

THURMAIR, M. Text, Texttypen, Textsorten. In: HELBIG, G. et al. (Hrsg.): Deutsch als 
Schwantuschke, F. - Motivationsschreiben

Fremdsprache. Ein internationales Handbuch. Berlin u.a., de Gruyter, 2001, 269-280.

VENOHR, E. Textmuster und Textsortenwissen aus der Sicht des Deutschen als Fremdsprache.Frankfurt am Main, Lang, 2007.

Recebido em 09/08/2014 aceito em 08/10/2014 